\title{
Miranda
}

Revue pluridisciplinaire du monde anglophone /

Multidisciplinary peer-reviewed journal on the English-

speaking world

13 | 2016

Thomas Spence and his Legacy: Bicentennial

Perspectives

\section{Pas de deux pour Shakespeare \\ "Mon cœur pour un sonnet" (Théâtre du Saulcy, Metz, 6 octobre 2016)}

Nathalie Vincent-Arnaud

\section{OpenEdition}

\section{Journals}

Édition électronique

URL : http://journals.openedition.org/miranda/9547

DOI : 10.4000/miranda. 9547

ISSN : 2108-6559

Éditeur

Université Toulouse - Jean Jaurès

Référence électronique

Nathalie Vincent-Arnaud, "Pas de deux pour Shakespeare

"Mon cœur pour un sonnet" (Théâtre du Saulcy, Metz, 6 octobre 2016)», Miranda [En ligne], 13| 2016,

mis en ligne le 24 novembre 2016, consulté le 16 février 2021. URL : http://journals.openedition.org/ miranda/9547 ; DOI : https://doi.org/10.4000/miranda.9547

Ce document a été généré automatiquement le 16 février 2021.

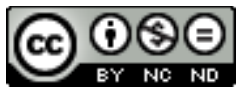

Miranda is licensed under a Creative Commons Attribution-NonCommercial-NoDerivatives 4.0 International License. 


\section{Pas de deux pour Shakespeare "Mon cœur pour un sonnet"} (Théâtre du Saulcy, Metz, 6 octobre 2016)

Nathalie Vincent-Arnaud

Je tiens à remercier Claire Bardelmann et Pierre Degott, ainsi qu'Aurélie Barré et Sébastien Amblard pour les échanges précieux que nous avons eus autour de leur spectacle ainsi que pour la photographie de Raymond Veber, jointe à ce compte rendu, qu'ils ont mise à ma disposition. 
1 Coproduction du Théâtre du Peuple de Bussang, de l'Opéra-Théâtre de MetzMétropole et du Théâtre du Saulcy (théâtre universitaire situé sur le campus du même nom et dirigé par Alain Billon), le spectacle "Mon cœur pour un sonnet", d'une durée d'une heure, réunit danse, musique, chant, comédie en un mélange subtil et contrasté, tissé de violences et d'accalmies, de tensions et d'attractions que n'aurait assurément pas renié le Barde. Le quadricentenaire de la mort de Shakespeare était l'occasion d'un tel projet, qui a d'abord vu le jour, durant l'été 2016, au Théâtre du Peuple de Bussang (dirigé par Vincent Goethals) avant de migrer sous les cieux messins pour servir d'interlude au colloque "Shakespeare et la musique en France, 19è-21è siècles" organisé les 6 et 7 octobre

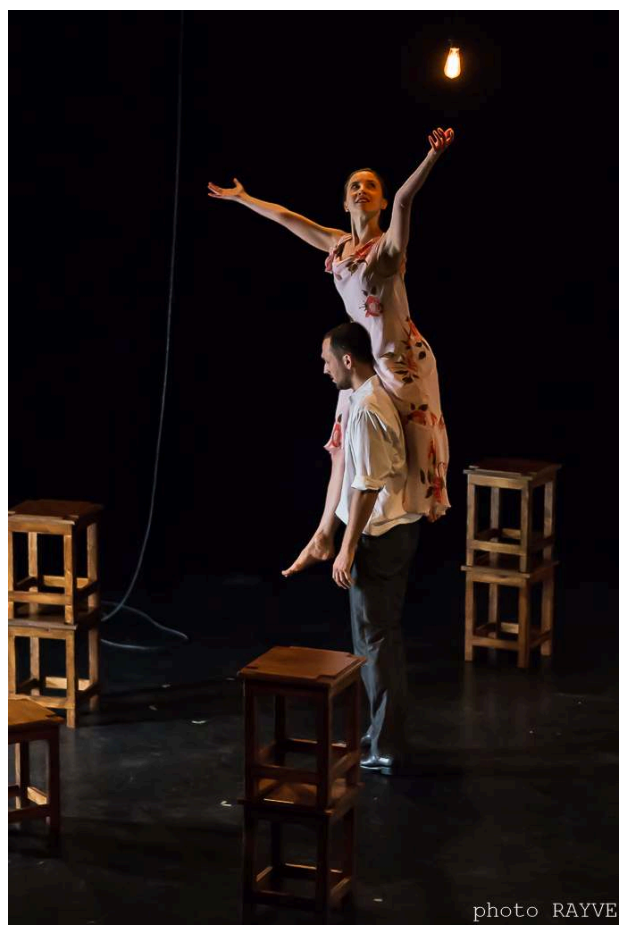
2016 à l'Université de Lorraine par Claire Bardelmann et Pierre Degott (http://ecritures.univ-lorraine.fr/sites/ecritures.univlorraine.fr/files/users/documents/colloque_shakespear_et_la_musique__programme.pdf). Le lendemain du spectacle, une table ronde dirigée par Francis Guinle (professeur à l'Université Lumière-Lyon 2 et spécialiste, entre autres, du théâtre élizabéthain et de la musique de la Renaissance), a permis aux deux artistes ainsi qu'au directeur artistique de l'Opéra-Théâtre de Metz-Métropole de dialoguer durant près d'une heure avec une partie du public de leur spectacle de la veille.

2 Le dialogue rapidement instauré entre Claire Bardelmann, enseignant-chercheur spécialiste de Shakespeare (et conseillère dramaturgique pour cette pièce), Aurélie Barré, danseuse à l'Opéra-Théâtre de Metz Métropole, et Sébastien Amblard, comédien, fut à l'origine de cette création en forme de spectacle total où les deux voix présentes sur scène sont habitées, d'un bout à l'autre, par une troisième voix: celle de la magistrale traduction des Sonnets par Robert Ellrodt, dont la configuration rythmique, les jeux sonores, la puissance évocatrice qu'elle parvient à recréer au fil des images s'accordent particulièrement bien à l'intensité des jeux scéniques déployés. Sur la vingtaine de sonnets que comporte la totalité du spectacle, le texte anglais s'invite toutefois à deux reprises - avec une très belle qualité de diction de la part des artistes pour faire entendre la musique originelle du vers shakespearien.

3 C'est dans un décor minimaliste constitué d'une dizaine de simples tabourets en bois qu'Aurélie Barré et Sébastien Amblard ont choisi d'évoluer et de donner corps à ces sonnets dont le potentiel dramatique et chorégraphique devient soudain évident pour le spectateur. La "force percutante" de la poésie de Shakespeare évoquée par Sébastien Amblard lors de la table ronde du lendemain trouve un écho à sa mesure à travers l'engagement corporel spectaculaire des deux artistes. Ces derniers deviennent, tour à tour ou simultanément, comédiens, danseurs, chanteurs, au fil d'échanges verbaux qui sont autant de changements de rôles, de postures, de tonalités illustrant les méandres 
des conceits et les variations thématiques parfois abruptes des sonnets. L'ensemble est renforcé par un jeu d'éclairages allant de la pénombre intimiste - quasi caravagesque aux pleins feux générés par les lampes latérales, cet ensemble d'oscillations et de gradations concourant, selon Aurélie Barré et Sébastien Amblard, à "réanimer la vie de deux personnages élizabéthains qui prennent corps" et à mettre en place l'histoire de ces deux personnages qui "se recherchent, se désirent", s'éprennent et se reprennent dans un continuum sans fin, dans une tension perpétuelle entre ombre et lumière.

Si l'ancrage dans l'époque élizabéthaine est clairement affiché, dès le début, par la robe noire caractéristique de l'époque Médicis dont le personnage féminin est revêtu, l'ensemble de la scénographie ne tarde guère à mettre en place une série de repères intemporels. Participent de cette évolution les costumes - de l'imposante parure Renaissance à la petite robe à fleurs, des chaussures rouges à hauts talons, emblèmes de l'objet du désir conquérant, aux pieds nus de la simplicité d'un corps et d'un esprit en quête de vérité - mais aussi les musiques : chants Renaissance, symphonie de Brahms, chanson de Dalida voisinent avec la comptine "Quand Fanny était un bébé", égrenée par une voix frêle de petite fille, pour évoquer, en contrepoint du texte shakespearien, les âges et les remous de la vie, les errances de l'amour, les vertiges de la connaissance de soi et de l'autre. L'intervention de voix off de jeunes enfants ${ }^{1}$ adressant des questions aux deux personnages en scène constitue l'un des points culminants du spectacle : tandis que, l'une après l'autre, les questions les plus saugrenues fusent (sur tous les thèmes imaginables illustrant les préoccupations enfantines habituelles), les réponses, au terme de très brefs silences désarmés, surgissent à leur tour sous forme de fragments de sonnets interrompus par l'arrivée d'une autre voix d'enfant, le tempo de l'ensemble ne cessant de se précipiter en un jeu de questions-réponses effréné. À l'effet comique résultant de cette juxtaposition de registres et de l'excellence du jeu d'Aurélie Barré et Sébastien Amblard, alternant postures figées et précipitation vocale et gestuelle soudaine, se mêle de manière obsédante le sentiment, rendu presque vertigineux par l'architecture même de la séquence, d'une œuvre passionnément ouverte, portant en elle, selon la formule des deux artistes, des "propositions infinies".

5 À ce périple musical et dialogique s'associe celui des tabourets, sans cesse réordonnés, transportés, empilés, escamotés, figurant autant de sièges, de supports, de tremplins pour l'envol de l'âme, de fenêtres ouvertes sur le monde. C'est sur l'espace scénique ainsi réorganisé et redimensionné au fil des séquences délimitées tout à la fois par les sonnets, les musiques et les voix extérieures, que se dessinent les déplacements en forme de marches, courses-poursuites, séries de pirouettes, de mouvements d'élévation en forme de développés et d'attitudes, de pas de deux où les corps se lient, se délient, se déportent, en un mouvement perpétuel d'élans, d'exaltations et de rétractions (tel ce baiser esquissé mais jamais complètement échangé, figure récurrente).

6 La chorégraphie de l'ensemble convoque pleinement le pouvoir métaphorique infini de la danse : le langage néo-classique superbement déployé par Aurélie Barré est avant tout au service d'une expressivité qui engage simultanément, ou à tour de rôle, les deux corps, chacun des deux abandonnant son étiquette première de danseur ou de comédien pour devenir tout à la fois l'un et l'autre. Un échange visant à la complétude que souligne efficacement le geste ultime du personnage masculin qui revêt le costume féminin Renaissance pour se figer dans la posture d'origine de la pièce. Cette réunion des époques, des genres, des antagonismes, achève de signer l'avènement de la discordia concors et de son corollaire : la perpétuation des questionnements et des vertiges que 
font surgir, à chaque nouvelle lecture, la "poésie foudroyante" (selon le mot des artistes) et la magie actualisatrice des Sonnets.

7 On ne peut que souhaiter à ce spectacle, tout aussi "foudroyant" et vertigineux, de voyager avec autant de bonheur et de succès hors des frontières lorraines et temporelles de cette année dédiée à Shakespeare.

\section{NOTES}

1. Les différentes voix off qui se font entendre au cours du spectacle sont celles de Glenn Campbell, Yves Thouvenel, Amandine, Camille et Élise.

INDEX

Thèmes : Dance

Keywords : dance, drama, music, Shakespeare, sonnet

Mots-clés : danse, musique, Shakespeare, sonnet, théâtre

\section{AUTEURS}

NATHALIE VINCENT-ARNAUD

Professeur

nathalie.vincent-arnaud@univ-tlse2.fr 